\title{
Redução da gestação gemelar em éguas puro sangue de corrida antes ou após a fixação uterina da vesícula embrionária*
}

\author{
Reduction of twin pregnancy in thoroughbred mares either before or after fixation
}

\begin{abstract}
Sandra Mara Fiala ${ }^{1}$, Cristina Rodrigues Trein ${ }^{1}$, Luciana Meirelles ${ }^{2}$, Eduardo Malschitzky ${ }^{1}$, Gustavo Winter ${ }^{2}$, Petra Garbade ${ }^{3}$, Ricardo Macedo Gregory ${ }^{1,3}$ \& Rodrigo Costa Mattos ${ }^{1,3}$
\end{abstract}

\begin{abstract}
RESUMO
A gestação gemelar é um sério problema devido às perdas econômicas geradas pelos altos índices de abortos, natimortos, mortalidade perinatal e queda de fertilidade da égua. Diversos métodos têm sido utilizados para realizar a redução de um dos gêmeos, como o esmagamento precoce de uma das vesículas sob controle ultra-sonográfico. O experimento teve como objetivo verificar a eficácia da redução de uma das vesículas embrionárias no $16^{\circ}$ dia pós-ovulação, antes ou depois de sua fixação no útero. Foram analisados os registros de 1337 éguas, da raça Puro Sangue de Corrida (PSC), pertencentes a três criatórios da região Sul do Brasil, num período de 10 anos. O diagnóstico de gestação foi realizado através de ultra-sonografia a partir do $10^{\circ}$ dia após a ovulação e repetido semanalmente até os 45 dias de gestação. Uma vez diagnosticadas gestações gemelares procedeu-se a eliminação de uma das vesículas embrionárias pelos seguintes métodos: (a) diagnóstico antes da fixação no $16^{\circ}$ dia pós-ovulação - esmagamento de uma das vesículas, (b) diagnóstico após o $16^{\circ}$ dia com vesículas não justapostas - esmagamento de uma das vesículas ou (c) diagnóstico após o $16^{\circ}$ dia com vesículas justapostas - restrição alimentar de concentrado durante duas semanas. A técnica foi bem sucedida quando ocorreu a redução de apenas uma das vesículas embrionárias. Naquelas gestações em que as vesículas se encontravam justapostas após os 30 dias optou-se pela administração de prostaglandina. A taxa geral de prenhez observada foi de 68,3\% (914/1337) e a porcentagem de gestação gemelar foi de 11,5\% (105/914). Diagnosticaram-se 38 gestações gemelares após a fixação, no $16^{\circ}$ e $62^{\circ}$ dia antes da fixação. Houve cinco perdas de dados. A redução embrionária realizada antes da fixação foi significativamente ( $\mathrm{p}=0,0317)$ mais bem sucedida do que quando o procedimento ocorreu após a fixação embrionária. Conclui-se, que o esmagamento de uma das vesículas é um procedimento eficaz na redução da gestação gemelar, principalmente quando realizado antes do $15^{\circ}$ dia de gestação.
\end{abstract}

Descritores: eqüinos, gestação gemelar, redução embrionária.

\begin{abstract}
Twin pregnacies in mares are detrimental due to high rates of abortion, stillborn, neonatal mortality, also contributing to a decrease in fertility rate. Therefore, some methods are used to eliminate one of the embrionyc vesicle. One of them is the manual compression of one embryonic vesicle controlled by ultrassonic imaging. The aim of this experiment was to determine the efficacy of embryo vesicle reduction, either before or after fixation, on Day 16, in mares. Records of 1337 thoroughbred mares belonging to three Southern Brazilian studs, during 10 years, were evaluated. Pregnancy was detected using rectal ultrasound starting Day 10 after ovulation ultrasound was repeated weekly until the Day 45 of pregnancy. When twin pregnancy was detected, one of the vesicles was eliminated using one of the following techniques: (a) diagnosis before fixation on day 16 - manual compression of one of the vesicles; (b) diagnosis after Day 16 and not adjacent vesicles - manual compression of one of the vesicles; (c) diagnosis after Day 16 and adjacent vesicles - dietary concentrate restriction for two weeks. The technique was considered well succeeded when just one of the vesicles was eliminated. Mares showing adjacent vesicles after Day 30 were treated with prostaglandin. The general pregnancy rate was 68.3\% (914/1337), $11.5 \%$ (105/914) being twin pregnancies. Regarding twin pregnancies, 38 were detected after fixation, on Days 16 , and 62 before fixation (missing data in 5 cases). Embryo crushing performed before fixation was significantly more efficacious $(p=0.0317)$ than after fixation. It was concluded that manual compression of one of the vesicles is an efficacious technique in reducing twin pregnancies, especially when performed before the Day 15 of pregnancy.
\end{abstract}

Key words: equine, twins, reduction.

\footnotetext{
* Artigo originado da Tese de Doutorado do primeiro autor. ${ }^{1}$ Programa de Pós-Graduação em Ciências Veterinárias (PPGCV)-UFRGS. ${ }^{2}$ Médico Veterinário autônomo. ${ }^{3}$ REPROLAB, Departamento de Medicina Animal, Faculdade de Veterinária-UFRGS.

CORRESPONDÊNCIA: R.C. Mattos [rcmattos@vortex.ufrgs.br ; FAX: +55 3316 7305].
} 


\section{INTRODUÇÃO}

Gêmeos eqüinos são originados geralmente de ovulações múltiplas [5], mais freqüentes no Puro Sangue de Corrida do que em outras raças.

A gestação gemelar é um sério problema na clínica equiina [11], causando perdas econômicas [8] devido aos altos índices de abortos, natimortos e mortalidade perinatal [14], bem como queda de fertilidade da égua após o aborto ou parto gemelar [16]. Registros históricos demonstram que a gestação gemelar responde por aproximadamente $40 \%$ das causas de aborto [12], sendo a morte fetal ocasionada, geralmente, por insuficiência placentária [1].

A égua, entretanto, possui mecanismos naturais de redução do número de conceptos, principalmente quando a fixação das vesículas embrionárias, no $16^{\circ}$ dia da gestação, ocorre unilateralmente [5].

Diversos métodos têm sido utilizados para realizar a redução de um dos gêmeos. A restrição alimentar por, aproximadamente, duas semanas foi preconizada por Merkt et al.[13]. O esmagamento precoce de uma das vesículas, através de controle ultrasonográfico [5], é técnica corrente utilizada nos haras e nas clínicas, com sucesso. Taxas de aproximadamente $90 \%$ de redução de um dos conceptos foram relatadas no Rio Grande do Sul $[2,10]$ quando os esmagamentos foram realizados entre o $15^{\circ} \mathrm{e}$ o $21^{\circ}$ dia de gestação.

O presente experimento teve como objetivo verificar a eficácia da redução de uma das vesículas embrionárias antes ou depois da fixação no $16^{\circ}$ dia.

\section{MATERIAIS E MÉTODOS}

Foram analisados os registros de 1337 éguas, da raça Puro Sangue de Corrida (PSC), pertencentes a três criatórios da região Sul do Brasil, num período de 10 anos. As éguas eram mantidas em campo nativo melhorado e a dieta suplementada diariamente com 2 a $3 \mathrm{Kg}$ de aveia esmagada. O controle da ovulação foi realizado quando as éguas se encontravam em estro, através de palpação retal e/ou ultra-sonografia e as coberturas foram efetuadas, após a visualização de um folículo de $35 \mathrm{~mm}$ de diâmetro, a cada 48 horas até o diagnóstico da ovulação.

O diagnóstico de gestação foi realizado através de ultra-sonografia a partir do $10^{\circ}$ dia após a ovulação e repetido semanalmente até 45 dias de gesta- ção. Quando foram diagnosticadas gestações gemelares, procedeu-se à eliminação de uma das vesículas embrionárias pelos seguintes métodos:

a) diagnóstico antes da fixação uterina no $16^{\circ}$ dia pós-ovulação - esmagamento de uma das vesículas [4];

b) diagnóstico após o $16^{\circ}$ dia pós-ovulação com vesículas não justapostas - esmagamento de uma das vesículas [4];

c) diagnóstico após o $16^{\circ}$ dia pós-ovulação com vesículas justapostas - restrição alimentar de concentrado durante duas semanas [13].

Após o procedimento de eliminação da vesícula embrionária, as éguas eram acompanhadas através de ultra-sonografia semanalmente para verificação da eficácia do tratamento. A técnica foi considerada bem sucedida quando ocorria a redução de apenas uma das vesículas embrionárias. Nas gestações em que as vesículas se encontravam justapostas após os 30 dias optou-se pela administração de prostaglandina.

As éguas, nas quais o procedimento realizado foi o esmagamento, foram divididas em dois grupos: $=15^{\circ}$ dia - éguas em que o procedimento foi realizado até $\mathrm{o} 15^{\circ}$ dia de gestação e $=16^{\circ}$ dia - quando o procedimento foi realizado a partir do $16^{\circ}$ dia até o $35^{\circ}$ dia.

$\mathrm{Na}$ análise estatística foram utilizadas a distribuição de freqüência e a análise de variância.

\section{RESULTADOS}

A taxa de prenhez foi de $68,3 \%$ (914/1337), enquanto que a porcentagem de gestação gemelar foi de 11,5\% (105/914). Das 105 gestações gemelares, foram avaliados 100 casos, tendo em vista que, em cinco deles (8\%), o procedimento adotado não foi registrado na ficha da égua. Diagnosticaram-se 38 gestações gemelares após a fixação uterina, no $16^{\circ}$ dia pós-ovulação e 62 antes da fixação. Das 38 gestações diagnosticadas após a fixação, $24(63,1 \%)$ ocorreram em cornos uterinos contralaterais. $\mathrm{O}$ esmagamento de uma das vesículas foi adotado em 86 casos (86\%), a restrição alimentar em 4 casos (4\%) e a administração de prostaglandina em 10 casos $(10 \%)$, devido à justaposição e à idade gestacional.

Naqueles casos em que se optou pela utilização de restrição alimentar por um período de duas semanas, devido à justaposição das vesículas embrionárias, observou-se sucesso em apenas um dos casos, sendo que nos demais $(75 \%)$ foi necessária a aplica- 
Fiala S.M., Trein C.R., Meirelles L., Malschitzky E., Winter G., Garbade P., Gregory R.M. \& Mattos R. C. Redução da gestação gemelar em éguas puro sangue de corrida antes ou após a fixação uterina da... Acta Scientiae Veterinariae. 31: 145 - 148.

ção de prostaglandina com a finalidade de interromper a gestação.

Verificou-se um maior número de vesículas esmagadas $(72,1 \%)$ no período $=15$ dia, enquanto que no $=16$ dia a porcentagem foi de $27,9 \%$, como observado na Tabela 1.

Tabela 1. Número de vesículas esmagadas de acordo com o momento do procedimento.

\begin{tabular}{ccc}
\hline Grupo & $\mathbf{n}$ & $\%$ \\
\hline$\geq 15^{\circ}$ dia & 62 & 72,1 \\
\hline$\geq 16^{\circ}$ dia & 24 & 27,9 \\
\hline
\end{tabular}

Na Tabela 2 pode-se observar a porcentagem de sucesso obtida nos 86 casos em que se utilizou o esmagamento de uma das vesículas.

Tabela 2. Índice de sucesso após a redução de uma das vesículas por esmagamento.

\begin{tabular}{ccc}
\hline Redução & $\mathbf{n}$ & $\%$ \\
\hline Sucesso & 82 & 95,3 \\
\hline Insucesso & 4 & 4,7 \\
\hline
\end{tabular}

Ao se analisar a Tabela 2, observa-se que houve sucesso em $95,3 \%$ dos procedimentos, demonstrando ser o esmagamento um método seguro de redução de uma das vesículas.

Na Tabela 3 estão expressos os índices de sucesso obtidos com os esmagamentos efetuados antes e após a fixação da vesícula embrionária

Tabela 3. Índice de sucesso de acordo com o dia do esmagamento.

\begin{tabular}{ccc}
\hline Grupo & $\mathbf{n}$ & Sucesso \\
\hline$\leq 15^{\circ}$ dia & 62 & $98,4 \%^{\mathrm{a}}$ \\
\hline$\leq 16^{\circ}$ dia & 24 & $87,5 \% \mathrm{~b}$ \\
\hline $\begin{array}{l}\text { Letras diferentes nas colunas } \\
(\mathrm{p}=0,0317) .\end{array}$
\end{tabular}

Ao se analisar a Tabela 3, observa-se que a redução embrionária realizada antes da fixação foi significativamente ( $\mathrm{p}=0,0317)$ mais bem sucedida do que quando o procedimento ocorreu após a fixação embrionária.

\section{DISCUSSÃO}

A taxa de prenhez foi de $68,3 \%$ (914/1337), enquanto que a porcentagem de gestação gemelar foi de $11,5 \%$ (105/914), inferior que os aproximadamente $15 \%$ observados anteriormente $[3,7,10]$.

Das 100 gestações gemelares avaliadas diagnosticaram-se 38 gestações gemelares após a fixação uterina, no $16^{\circ}$ dia pós-ovulação e 62 antes da fixação. Das 38 gestações diagnosticadas após a fixação, $24(63,1 \%)$ ocorreram em cornos uterinos contralaterais, o que discorda do descrito na literatura [6], que relata a ocorrência de apenas $30 \%$ de fixação em cornos diferentes.

Naqueles casos em que se optou pela utilização de restrição alimentar por um período de duas semanas, devido à justaposição das vesículas embrionárias, observou-se sucesso em apenas um dos casos, sendo que nos demais $(75 \%)$ foi necessária a aplicação de prostaglandina com a finalidade de interromper a gestação. Os dados diferem dos resultados que obtiveram sucesso em aproximadamente $65 \%$ dos casos [13]. Provavelmente, este menor índice de sucesso deva-se a diferenças de dieta alimentar e de condição corporal entre as éguas da Alemanha e do Brasil. Enquanto nas éguas alemãs a alimentação é baseada em concentrado e feno de alfafa e a condição corporal [9] média é 4,5, as éguas do presente trabalho apresentavam condição corporal média 3,5 e sua nutrição era baseada em campo nativo melhorado com pequena suplementação de concentrado. Com estas condições, é provável que a restrição alimentar tenha sido muito pequena para promover a eliminação de uma das vesículas embrionárias.

O sucesso em $95,3 \%$ dos procedimentos, demonstra ser o esmagamento um método seguro de redução de uma das vesículas, confirmando achados anteriores $[2,10]$. Os resultados obtidos sugerem que a liberação de prostaglandina F2a, que ocorre durante mais de 90 minutos após o esmagamento [6], não é suficiente para provocar danos ao corpo lúteo que impeçam a continuidade da gestação. 
Fiala S.M., Trein C.R., Meirelles L., Malschitzky E., Winter G., Garbade P., Gregory R.M. \& Mattos R. C. Redução da gestação gemelar em éguas puro sangue de corrida antes ou após a fixação uterina da... Acta Scientiae Veterinariae. $31: 145$ - 148.

A redução embrionária realizada antes da fixação foi significativamente mais bem sucedida do que quando o procedimento ocorreu após a fixação embrionária. Os resultados concordam com os de Alda et al. [2], que observaram menor sucesso do esmagamento após o $20^{\circ}$ dia de gestação e não concordam com os de Pascoe et al. [15]. No entanto, observa-se que, já a partir do $16^{\circ}$ dia de gestação, a manipulação ou a quantidade de líquido, liberada no lúmen uterino pro- veniente da vesícula esmagada, provavelmente promovem a liberação de prostaglandina, o que provocaria a lise do corpo lúteo.

\section{CONCLUSÃO}

Conclui-se que o esmagamento de uma das vesículas embrionárias é um procedimento eficaz na redução da gestação gemelar, principalmente quando utilizado até $o 15^{\circ}$ dia de gestação.

\section{REFERÊNCIAS}

1 Acland H. M. 1993. Abortion in mares. In:. Mckinnon A. O \& Voss J. L.(Eds). Equine Reproduction. Philadelphia: Lea \& Febiger, pp. 554-562.

2 Alda J. L., Depra N. M., Bergamo P N. L., Brass K. E., De la Corte F. D. \& Silva C. A. M. 1995. Redução da prenhez gemelar na égua por esmagamento. Arquivos da Faculdade de Veterinária UFRGS. 23: 19-25.

3 Bowman T. 1986. Ultrassonic diagnosis and management of early twins in the mare. In: Proceedings of the American Association of Equine Practitioners (Nashville, USA). 32: 35-43.

4 Ginther O. J. 1986. Ultrasonic imaging and reproductive events in the mare. Cross Plains: Equiservices Publishing, $378 \mathrm{p}$.

5 Ginther O. J. 1989. Twin embryos in mares. II: Post fixation embryo reduction. Equine Veterinary Journal. 21: 171-174.

6 Ginther O. J. 1992. Reproductive Biology of the Mare: Basic and applied aspects. 2nd edn. Cross Plains: Equiservices Publishing, $642 \mathrm{p}$.

7 Kahn W. 1991. Atlas und Lehrbuch der Ultraschalldiagnostik. Hannover: Schültersche, p. 25.

8 MacPherson M. L. \& Reimer J. M. 2000. Twin reduction in the mare: current options. Animal Reproduction Science. 60-61:233-244.

9 Malschitzky E. 1998. Efeito de diferentes tratamentos pós-cobertura na fertilidade de éguas Puro-Sangue de Corrida. $74 \mathrm{f}$. Porto Alegre, RS. Dissertação (Mestrado em Ciências Veterinárias) - Programa de Pós-Graduação em Ciências Veterinárias, Universidade Federal do Rio Grande do Sul.

10 Mattos R. C., Zimmer Jr. O., Beck P. \& Gregory R. M. 1994. Gestação gemelar na égua. Possibilidades de redução do número de embriões. Arquivos da Faculdade de Veterinária UFRGS. 22: 19-23.

11 Meier H. P., Gertsch U. \& Kohler U. 1997. The spontaneous reduction of a twin pregnancy in two mares. Schweizer Archives Tierheilkdunde. 139: 71-77.

12 Merkt H. 1985. Trächtigkeitsverluste beim Pferd und die Möglichkeiten ihrer Reduzierung. Tierärztliche Umschau. 40: 428-435

13 Merkt H., Jungnickel S. \& Klug E. 1982. Reduction of early twin pregnancy to single pregnancy in the mare by dietetic means. Journal of Reproduction and Fertility.32 (Suppl): 451-452.

14 Miller A. \& Woods G. L. 1988. Diagnosis and correction of twin pregnancy in the mare. Veterinary Clinics of North America: Equine Practice. 4: 215-220.

15 Pascoe D. R., Pascoe R. R., Hughes J. P., Stanbenfeldt G. H. \& Kindahl H. 1987. Comparison of two techniques and three therapies for management of twin conceptuses by manual embryonic reduction. Journal of Reproduction and Fertility. 35 (Suppl): 701-702.

16 Pascoe R. R. 1983. Methods for the treatment of twin pregnancy in the mare. Equine Veterinary Journal. 15: 40-42. 